\title{
CDCT: CT Scan Images based on Mechanism for Lung Cancer Detection
}

\author{
Sakshi Sharma, Maninder Pal Singh, Baljeet Kaur Nagra
}

\begin{abstract}
Image classification is one of the major issues of image pre-processing approach. To resolve this issue a large number of classification approaches has been developed. In this work, a novel SVM-FA (support vector machine optimized with firefly approach) classifier is developed for detecting the lung cancer on the basis of the CT images. Lung cancer is considered one of the most critical and vital. Thus the early analysis of such kind of disease is required. For this purpose, the study implements the image pre-processing (filtration and segmentation) techniques to the input CT scan images. Then the SVM classifier, optimized with firefly approach is applied to the pre-processed data. The target of the work is to enhance the accuracy in the final prediction or output. For evaluating the proficiency level of the proposed SVM-FA approach, a comparison analysis is also performed in this work. The comparison is done among proposed work, traditional work and SVM classifier. On the basis of the obtained facts and figures, the proposed work is found to be effective and efficient in terms of the accuracy (96\%) and specificity (83.333\%) respectively.
\end{abstract}

Index Terms: Medical image pre-processing, SVM classifier, Firefly optimization, Lung cancer.

\section{INTRODUCTION}

A tumor is an anomalous growth of new tissue and that can be formed in any organs of human body. There are various kinds of cancer like lung, bone and brain cancer. Nowadays, this cancerous is considered to be biggest danger and vital cancer in this world, including smallest diagnosis survival rate [1-2].

Lung cancer is a condition in which cells replicates uncontrollably and grows into a tumor. It may be possible to take away the cancer cell via alveolus in the hemoglobin or in the lymphatic in which lung tissue is surrounded. The lymph flows into the lymphatic vessels and flows to the lymphatic gland placed in both the alveolus and in the chest's middle. As lymph flows from lungs to the center of the chest, the tumor of lung cancer grows towards the chest. Metastasis takes place if tumor unit abscond the place whereabouts it started and goes to a lymphatic unit or other part of body via the blood stream [2]. Cancerous, in which tumor is began to grow into alveolus which is known as initial cancerous. Further, this cancer is classified into 2 major stages: small cell and non-small cell lung cancer which is further subcategorized as: Carcinoma, Aden carcinoma and Squamous cell carcinomas. In 2008, amongst Jordanians, the

Revised Manuscript Received on July 22, 2019

Sakshi Sharma, CSE, Chandigarh University, Mohali, India.

Maninder Pal Singh, CSE, Chandigarh University, Mohali, India,

Baljeet Kaur Nagra, CSE, Chandigarh University, Mohali, India. order of classification of cancers for both males and females observed more than 300 cases of

cancerous that represent $(7.7 \%)$ of all cancer cases freshly predicted in the same year. 297 i.e. almost $13.1 \%$ of males and 59 females i.e. $2.5 \%$ were diagnosed with lung cancer. This showed a ratio of male-female i.e. 5:1 and thus, this cancer is ranked second among males and tenth among females [3].Generally, there are four stages to detect lung cancer. Firstly, normal and abnormal CT images are collected by accessible database from the IMBA web page [5]. In the second stage, some image enhancement methodologies are implemented in order to acquire high quality and clear images. Then, algorithms of image segmentation are applied. These algorithms have significant role in the image processing and in the last stage, the general characteristics are extracted from an improved segmented image indicating normal or abnormal images.

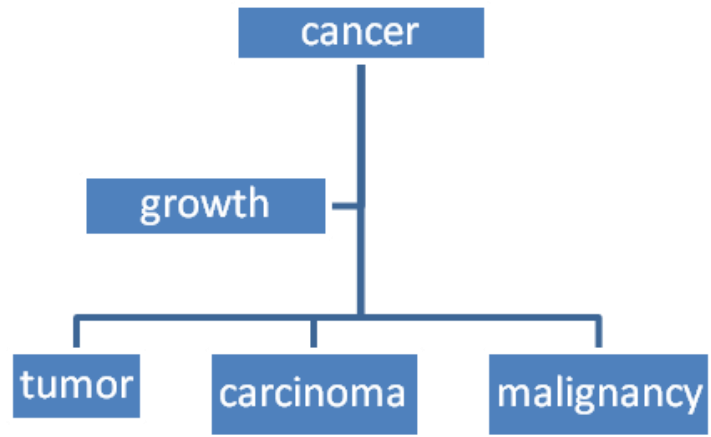

Fig. 1. Lung Cancer block diagram

\section{RELATED WORK}

Asuntha.A, et.al[1] After using edge detection, segmentation, filtering some features are extracted from the images .At the end, they projected a model to extracted the features to reduce the obstacle and isolate the desired shape of images.

Mokhled Altarawneh[2] proposed a detected features for accurate image pixels and detectable because quality and accuracy plays a major role in this research to maintain the stability of images.

Mokhled S. Al-Tarawneh [5] projected a model to cancerous diagnose having usage of Image Processing techniques. With this method, images were denoised using Gabor filter. Gabor filter provided the best results. Two segmentation methods were used for segmentation purpose. It followed the algorithms marker-controlled watershed segmentation and thresholding approach. In

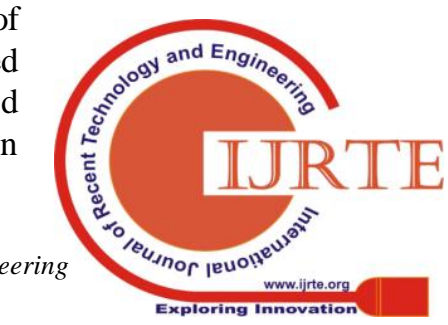




\section{CDCT: CT Scan images based on mechanism for Lung Cancer Detection}

contrast to thresholding approach, Marker-controlled segmentation technique had enhanced result. Feature extraction of image is performed via binarization and masking approach was used to identify cancer.

Anita chaudhary [6] proposed a method for detecting the cancerous by processing the scanned pictures. This model utilized Gabor filter for reducing the level of noise. Marker-controlled watershed and threshold segmentation were used to segment the images. Tumor is identified by extracting the features such as Area, perimeter, and roundness.

The authors Nooshin Hadavi and Md. Jan Nordin [7] have developed a method for diagnosing of CT-Scan images dependent on CLA (Cellular Learning Automata). Gabor filter was utilized for removal of noise in the input image. Segmentation was done through Region growing algorithm. Thus from the segments of the image, the process feature extraction is performed and these feature are applied to the new algorithm cellular automata to identify cancer.

Aggarwal, T., et al [8] have proposed a model in which he utilized gray level characteristics and optimum threshold for segmentation and geometrical features were extracted and LDA (linear discriminate analysis) classifier was used to differentiate nodule and pulmonary structure. Although, it used simple techniques of image processing and thus this model offered $84 \%$ accuracy and $53.33 \%$ specificity and $97.14 \%$ sensitivity.

\section{PROPOSED WORK}

Medical image processing is one of the prominent topics that is considered for the research work. In medical domain, the image processing is used for the purpose of diagnosing the diseases on the basis of various pictures i.e. MRI, CT scan, x-ray etc. Before diagnosing the final disease form the images, various pre-processing techniques are applied. These techniques are like extraction and classification features etc. Based on the review, it is analyzed that the traditional work done for lung cancer detection by using ct scan perform the cancer diagnosis by using SVM Gabor filters, watershed segmentation technique and SVM classification approach. On the basis of the obtained results, the traditional approach is found quite effective but still more correction are possible to enhance the accuracy level of the obtained results. Thus the backlog of the traditional work is factors used by SVM classifiers for classification. Thus, a novel approach of classification is required to be developed.

In order to overcome the backlog of traditional work, the present work applies the image pre-processing approaches and SVM classification approach. In proposed work, the factors of SVM classifier is optimized by using the firefly optimization approach. In order to perform improvisations in SVM, the value of sigma and bounding box boundaries factor has been changed from 1 . But the variation of these factors is not an easy task. Thus to perform the variations in factors, the concept of firefly optimization technique is applied in this work. Following (figure 2) is flowchart of the proposed work.

\section{Start}

2. Next step is to gather the data related to the lung cancer. The collected data is in the form of the images. These images act as a input images for proposed SVM-FA approach.

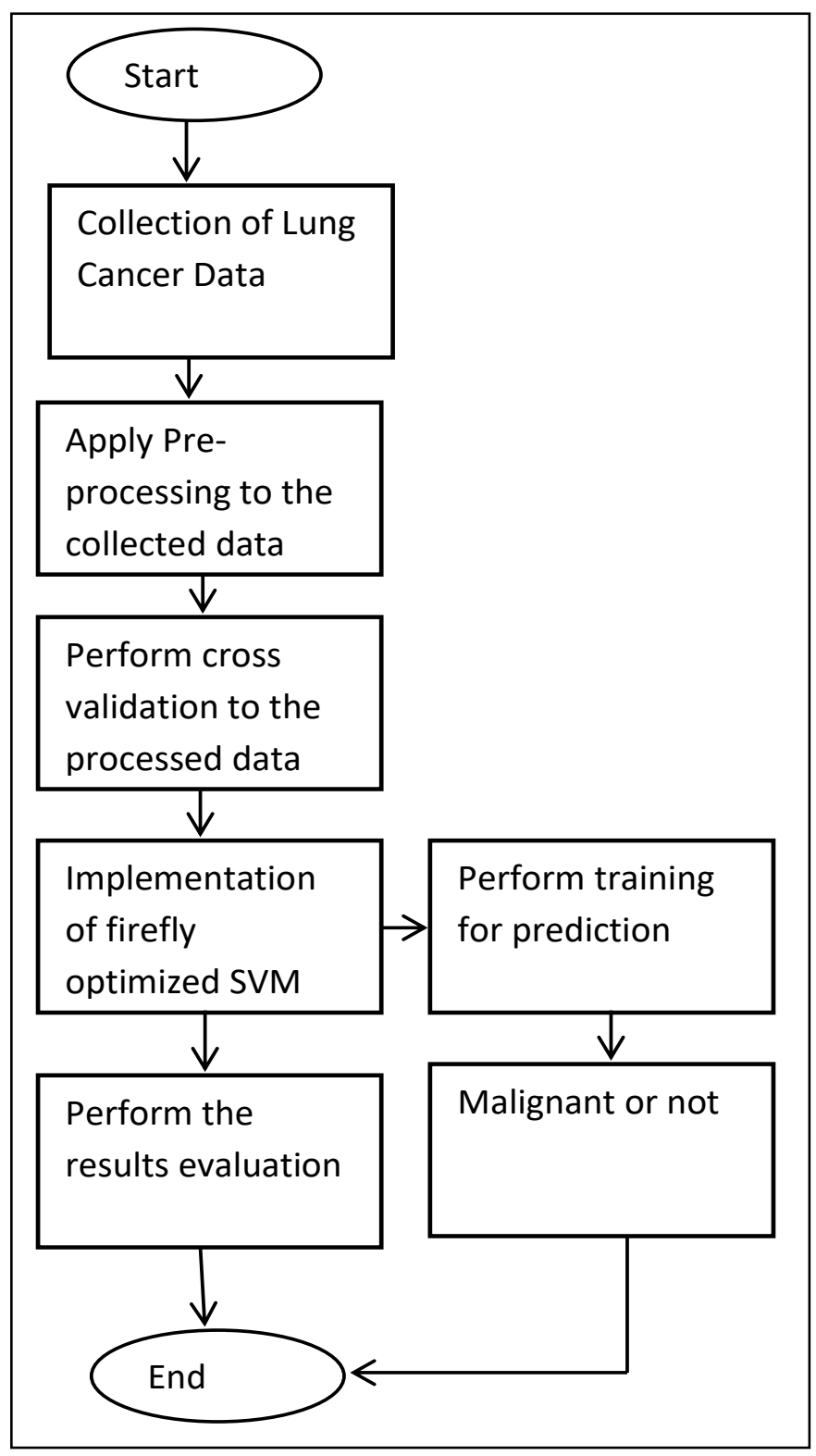

Fig. 2. Framework of proposed work

3. After getting the input images, next step is to apply the image pre-processing approach in pre-processing the filtration and segmentation approach is applied and then the cross validation is performed to the pre-processed images.

4. In this step, the SVM classification approach is optimized by using the firefly approach.

5. Next step is to perform training on the input data. The training is performed with the perspective of the prediction.

6. After training of the data, it is evaluated whether the malignant is detected or not. Here the lung cancer

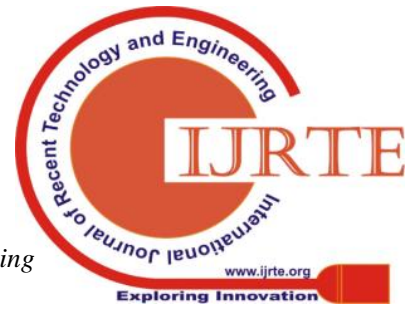


is detected in the form of malignant.

7. End

\section{IMAGE CLASSIFICATION AND OPTIMIZATION}

\section{A. SVM}

Image classification is one of standard concerns in image

processing. The classification of images aims to foresee the order of initial pictures applying its characteristics. To solve this issue, Adaptive boost (Ad boosted) KNN, ANN, SVM are some of the methodologies which can be used.

SVM is the significant approach in both pattern image classifications. The design of SVM is made in order to divide an arranged training pictures into several stages, $\left(\mathrm{x}_{1}, \mathrm{y}_{1}\right),\left(\mathrm{x}_{2}\right.$, $\left.\mathrm{y}_{2}\right), \ldots,\left(\mathrm{x}_{\mathrm{n}}, \mathrm{y}_{\mathrm{n}}\right)$ where $\mathrm{x}_{\mathrm{i}}$ in $\mathrm{R}^{\mathrm{d}}, \mathrm{d}$-dimensional feature space, and $y_{i}$ in $\{-1,+1\}$, the class label, with $i=1$..n [1]. It develops the optimum splitting hyper planes on the basis of a kernel function (K). When the classified points are divided by hyper plane, those images refer to class -1 and other one refer to class+1 [9].

\section{B.Firefly Optimization}

Firefly algorithm comprises of two variables, namely, the intensity of light and attractiveness. They get attracted to another one with a flash shiner than her. Thus, both factors work simultaneously.

Therefore, attractiveness and the specific distance $r$ via source of light vary inversely. Consequently, increase in the distance would be decrease the light and attractiveness.

The pseudo code for firefly algorithm is as follows:

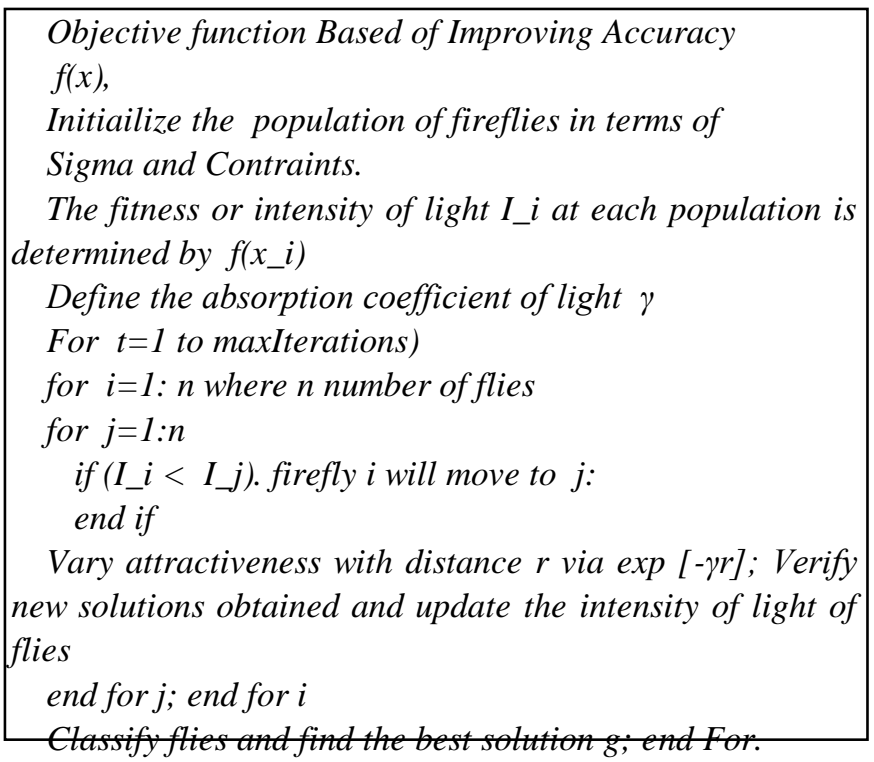

\section{V.RESULT ANALYSIS}

This work implements the firefly optimized SVM approach for lung cancer detection from the medical images. The results evaluated from the implementation of proposed image processing mechanism for detecting the lung cancer is shown in this region.

The execution of the proposed work is calculated in the terms of accuracy, sensitivity and specificity as shown in following graphs. A comparison analysis among traditional work, SVM approach and proposed SVM approach is also processed in order to prove the proposed work by the proficiency.

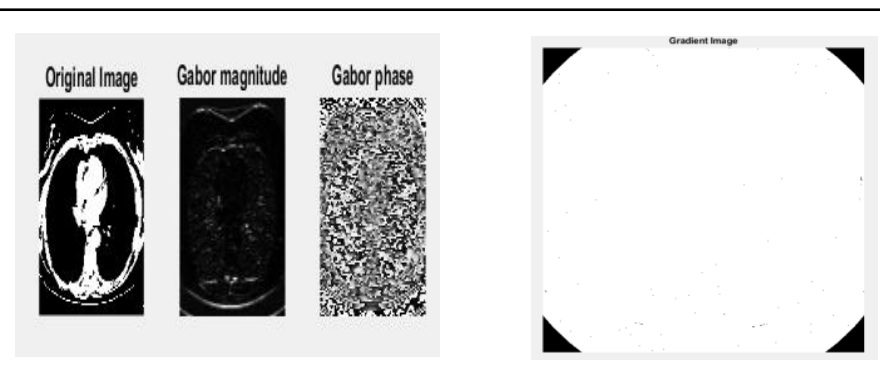

Fig 3a. Original Image with

Fig 3b. Gradient Image gabor filter
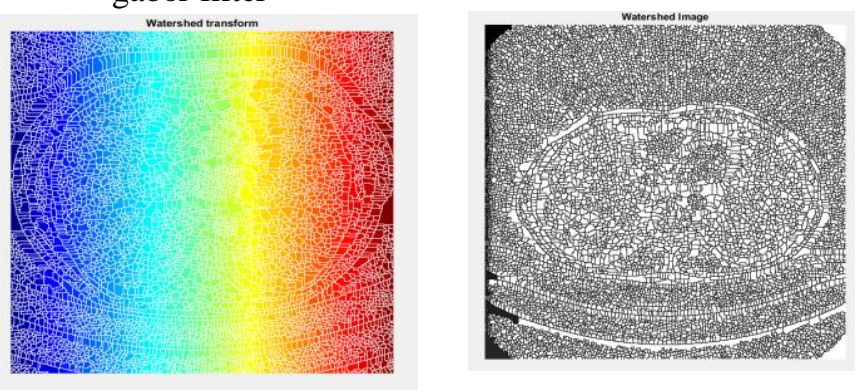

Fig 3c. Watershed Transform Fig 3d. Watershed Image
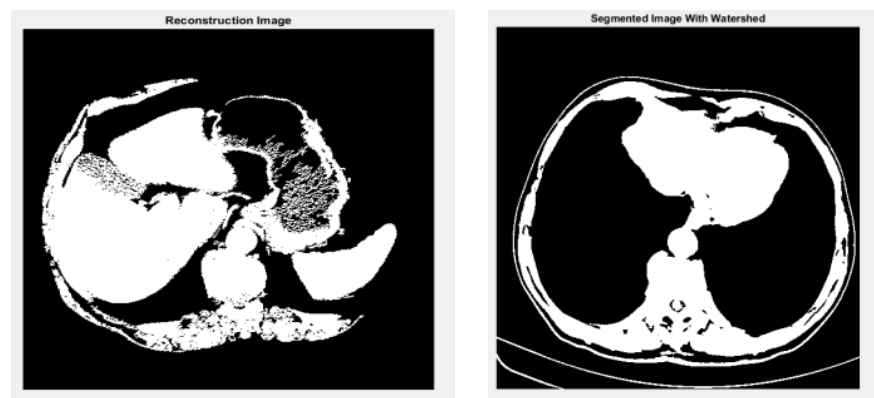

Fig 3e. Reconstruction Image Fig 3f. Segmented Image with Watershed

Fig. 3. Various steps in image processing

Figure 5 represents the graphical comparison analysis of traditional, SVM and proposed SVM approach in the terms of accuracy. Following is the formulation for evaluating the accuracy of the proposed approach.

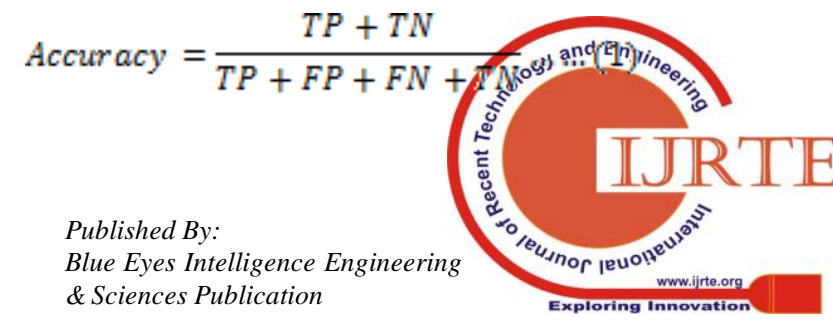




\section{CDCT: CT Scan images based on mechanism for Lung Cancer Detection}

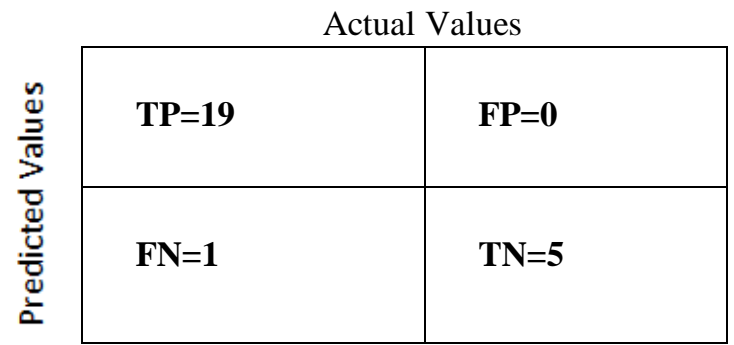

Fig. 4. Confusion Matrix

This $\mathrm{x}$ axis in the graph shows the performance of various classifiers and the y axis calibrates the data for the accuracy and it ranges from 0 to 100 .

On the basis of the observed output, it is proven that accuracy percentage of the proposed SVM approach is highest in comparison to the traditional approaches.

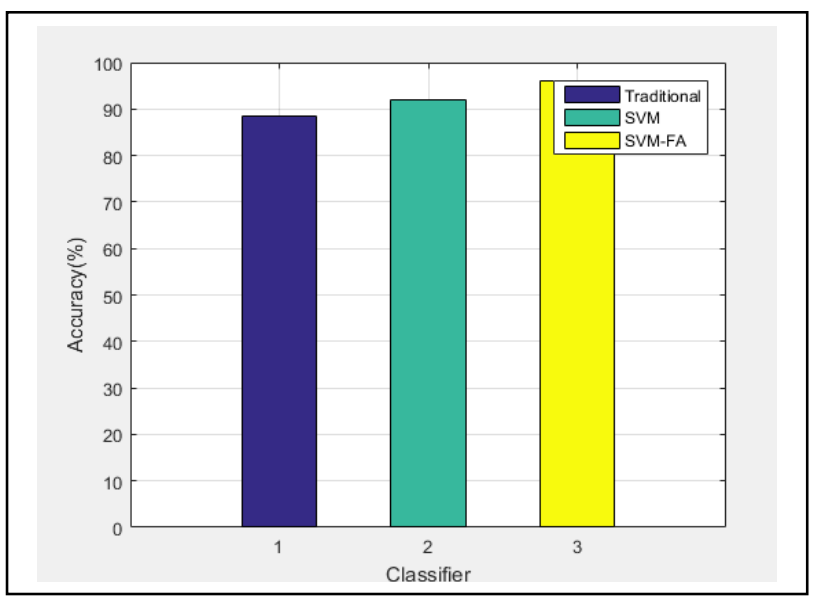

Fig. 5. Comparison analysis of traditional, SVM and proposed approach with respect to the accuracy (\%)

The sensitivity of the proposed and traditional classifiers is shown in graph of figure 6 . The sensitivity is evaluated as below.

$$
\text { Sensitivity }=\frac{T P}{T P+F N} \ldots \ldots(
$$

The chart in fig. 6 defines the sensitivity of traditional, SVM as well as proposed SVM approach. The observations from the graph delineates that the sensitivity of all of the classification approach is similar and higher i.e. $100 \%$.

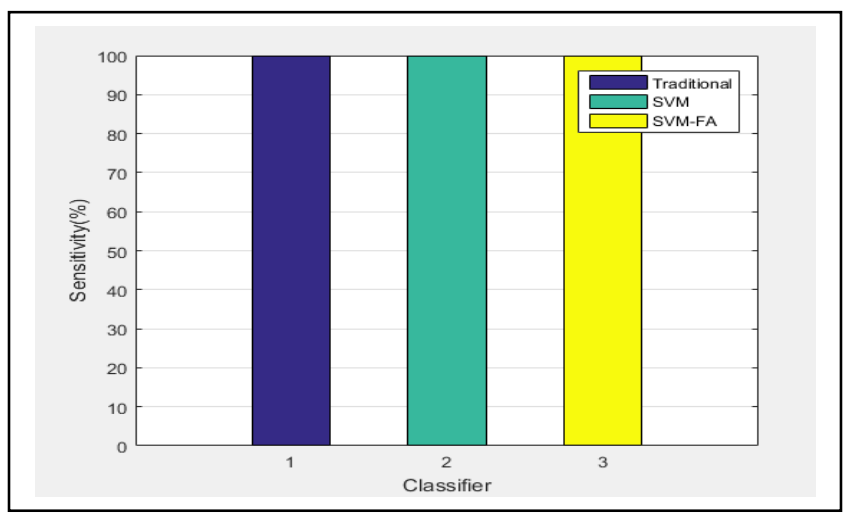

Fig. 6. Comparison analysis of traditional, SVM and proposed approach with respect to the sensitivity (\%)

The graph in figure 7 delineates the comparison analysis of specificity of traditional classifier, SVM classifier and proposed firefly-SVM optimized classifier. The specificity of proposed classifier is found to be higher in comparison to the traditional and SVM classification approach. The following equation is applied for evaluating the specificity of the classifiers.

$$
\text { Specificity }=\frac{T N}{F P+T N} \ldots \ldots(3)
$$

The observed specificity of proposed work is $83.333 \%$, for SVM classifier is $50 \%$ and for traditional classifier it is $40 \%$. The higher specificity proves the efficiency and effectiveness of the work.

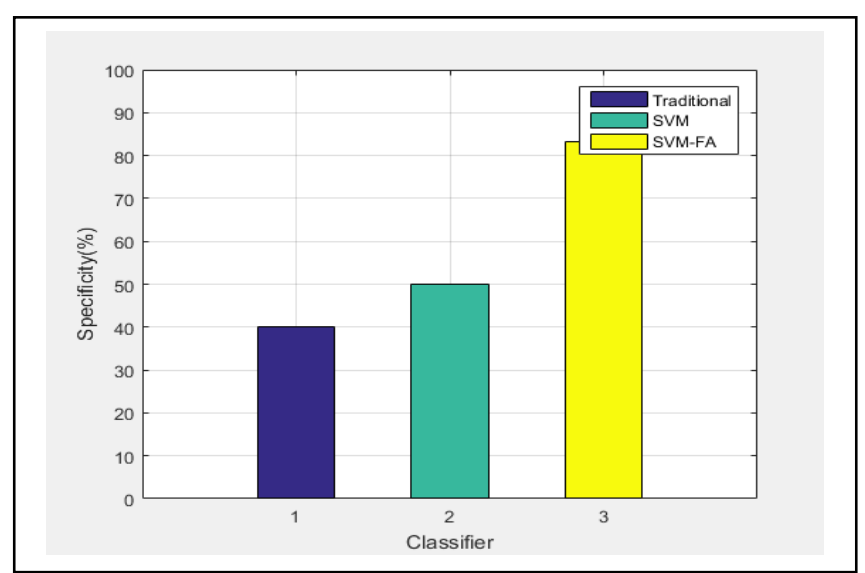

Fig. 7. Comparison analysis of traditional, SVM and proposed approach with respect to the specificity (\%)

The facts and figures observed from above defined graphs is calibrated in table 1 . The values of the table depicts that the proposed SVM-FA classifier has the highest accuracy (96\%), than SVM classifier (92\%) and traditional classifier (88.4\%).

Similarly, the specificity of the proposed approach is $83.33 \%$ that is higher than the traditional and SVM approach i.e. $40 \%$ and $50 \%$. Whereas, the sensitivity of three of the considered classifiers are same i.e. $100 \%$.

Table 1 Comparison analysis of traditional; classifier, SVM classifier and SVM-FA classifier

\begin{tabular}{|c|c|c|c|}
\hline Classifiers & Accuracy & Sensitivity & Specificity \\
\hline $\begin{array}{c}\text { Traditional } \\
\text { Classifier }\end{array}$ & 88.4 & 100 & 40 \\
\hline
\end{tabular}

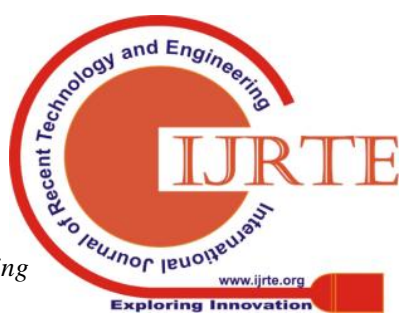




\begin{tabular}{|c|c|c|c|}
\hline $\begin{array}{c}\text { SVM } \\
\text { classifier }\end{array}$ & 92 & 100 & 50 \\
\hline $\begin{array}{c}\text { SVM-FA } \\
\text { classifier }\end{array}$ & 96 & 100 & 83.333 \\
\hline
\end{tabular}

\section{CONCLUSION AND FUTURE SCOPE}

Now days, the images are used in each and every domain for the purpose of detection and analysis. Medical image processing is one of the domains where the diseases are diagnosed based on different images such as CT scan, MRI, $\mathrm{X}$-ray etc. In this work, a novel approach is developed for detecting the lung cancer by using CT scans. The SVM classifier is optimized by using firefly optimization approach. The performance of the proposed work is considered preferably with efficiency, specificity also sensitivity. The comparison output views the proposed workout performs the traditional classifiers and SVM classifiers with respect to the considered performance metrics.

In future the execution of the expected work can be improved in many forms like the work could be done to reduce the complexity. Along with this, more amendments are possible on feature selection and extraction mechanisms.

\section{REFERENCES}

1. Asuntha, A., Banu, P. A., Ainthaviarasi, K., Kumar, B. S., \& Srinivasan, A. (2017). Feature extraction to detect Bone Cancer Using Image Processing. Research Journal of Pharmaceutical Biological and Chemical Sciences, $8(3), 434-442$.

2. Non-Small Cell Lung Cancer, Available at: http://www.katemacintyrefoundation.org/pdf/non-small-cell.pdf, Adapted from National Cancer Institute (NCI) and Patients Livingith Cancer (PLWC), 2007, (accessed July 2011).

3. Tarawneh, M., Nimri, O., Arqoub, K., \& Zaghal, M. (2007). Cancer incidence in Jordan. Jordan National Cancer Registry.

4. Lung Cancer Database, Available at: https://eddie.via.cornell.edu/cgibin/datac/signon.cgi, (accessed July 2011).

5. Al-Tarawneh, M. S. (2012). Lung cancer detection using image processing techniques. Leonardo Electronic Journal of Practices and Technologies, $11(21), 147-58$

6. Chaudhary, A., \& Singh, S. S. (2012, September). Lung cancer detection on CT images by using image processing. In 2012 International Conference on Computing Sciences (pp. 142-146). IEEE.

7. Hadavi, N., Nordin, M. J., \& Shojaeipour, A. (2014, June). Lung cancer diagnosis using CT-scan images based on cellular learning automata. In 2014 International Conference on Computer and Information Sciences (ICCOINS) (pp. 1-5). IEEE.

8. Aggarwal, T., Furqan, A., \& Kalra, K. (2015, August). Feature extraction and LDA based classification of lung nodules in chest CT scan images. In 2015 International Conference on Advances in Computing, Communications and Informatics (ICACCI) (pp. 1189-1193). IEEE.

9. Thai, L. H., Hai, T. S., \& Thuy, N. T. (2012). Image classification using support vector machine and artificial neural network. International Journal of Information Technology and Computer Science (IJITCS), 4(5), 32-38.

10. Makaju, S., Prasad, P. W. C., Alsadoon, A., Singh, A. K., \& Elchouemi, A. (2018). Lung cancer detection using CT scan images. Procedia Computer Science, 125, 107-114

11. Mesleh, A. M. (2017). Lung Cancer Detection Using Multi-Layer Neural Networks with Independent Component Analysis: A Comparative Study of Training Algorithms. Jordan Journal of Biological Sciences, 10(4).

12. Jin, X. Y.,Zhang, Y. C., \& Jin, Q. L. (2016, December). Pulmonary nodule detection based on CT images using convolution neural network. In 2016 9th International Symposium on Computational Intelligence and Design (ISCID) (Vol. 1, pp. 202-204). IEEE.

13. Dwivedi, M. S. A., Borse, M. R., \& Yametkar, M. A. M. (2014). Lung Cancer detection and Classification by using Machine Learning \&
Multinomial Bayesian. IOSR Journal of Electronics and Communication Engineering (IOSR-JECE), 9(1), 69-75

14. Baswade, A. M., Joshi, K. D., \& Nalwade, P. S. (2012). A Comparative Study Of K-Means and Weighted K-Means for Clustering. International Journal of Engineering Research \& Technology, 1(10).

15. Khobragade, S., Tiwari, A., Patil, C. Y., \& Narke, V. (2016, July) Automatic detection of major lung diseases using Chest Radiographs and classification by feed-forward artificial neural network. In 2016 IEEE 1st International Conference on Power Electronics, Intelligent Control and Energy Systems (ICPEICES) (pp. 1-5). IEEE.

16. Sevani, A., Modi, H., Patel, S., \& Patel, H. (2018). Implementation of Image Processing Techniques for Identifying Different Stages of Lung Cancer. International Journal of Applied Engineering Research, 13(8), 6493-6499.

17. Ignatious, S., Joseph, R., John, J., \& Prahladan, A. (2010). Computer Aided Lung Cancer Detection and Tumor Staging in CT image using Image Processing. International Journal of Computer Applications, 975, 8887

18. Tariq, A., Akram, M. U., \& Javed, M. Y. (2013, April). Lung nodule detection in CT images using neuro fuzzy classifier. In 2013 Fourth International Workshop on Computational Intelligence in Medical Imaging (CIMI) (pp. 49-53). IEEE

19. Vas, M., \& Dessai, A. (2017, August). Lung cancer detection system using lung CT image processing. In 2017 International Conference on Computing, Communication, Control and Automation (ICCUBEA) (pp. 1-5). IEEE.

20. Miah, M. B. A., \& Yousuf, M. A. (2015, May). Detection of lung cancer from CT image using image processing and neural network. In 2015 International conference on electrical engineering and information communication technology (ICEEICT) (pp. 1-6). ieee.

21. Anifah, L., Harimurti, R., Permatasari, Z., Rusimamto, P. W., \& Muhamad, A. R. (2017, October). Cancer lungs detection on CT scan image using artificial neural network backpropagation based gray level coocurrence matrices feature. In 2017 International Conference on Advanced Computer Science and Information Systems (ICACSIS) (pp. 327-332). IEEE

22. Than, J. C., Saba, L., Noor, N. M., Rijal, O. M., Kassim, R. M., Yunus, A., ... \& Suri, J. S. (2017). Lung disease stratification using amalgamation of Riesz and Gabor transforms in machine learning framework. Computers in biology and medicine, 89, 197-211.

\section{AUTHORS PROFILE}

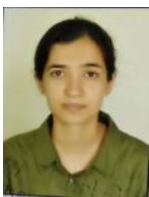

Sakshi Sharma currently pursuing M.tech from Chandigarh University and B.tech from Eternal University.

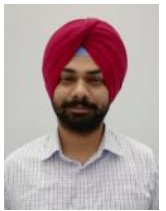

Maninder Pal Singh currently pursuing $\mathrm{PhD}$ from Chandigarh University, M.tech from LPU, B.tech from PTU and member of IEEE COMSOC.

Baljeet Kaur Nagra Research Analyst in Chandigarh University, M.tech from CGC Landran, B.tech from Swami Devi Dyal Institute of Engineering and Technology Barwala. 